\title{
Perception and Behavior of InsBot : Robot-Animal Interaction Issues
}

\author{
Fabien Tâche, Masoud Asadpour, Gilles Caprari, Walter Karlen, Roland Siegwart \\ Autonomous Systems Lab (http://asl.epfl.ch) \\ Ecole Polytechnique Fédérale de Lausanne (EPFL) \\ CH-1015 Lausanne, Switzerland \\ Email: \{fabien.tache, masoud.asadpour, gilles.caprari, walter.karlen, roland.siegwart\}@epfl.ch
}

\begin{abstract}
This paper describes the hardware and behavior implementation of a miniature robot, in size of a match box, that is able to interact with cockroaches. The robot is equipped with two micro-processors dedicated to hardware processing and behavior generation. It is also equipped with 12 infra-red proximity sensors, 2 light sensors, a linear camera and a battery that allows 3 hours autonomy. The robot can discriminate cockroaches, other robots, environment boundaries and shelters. It has also three means of communication: a wireless module for monitoring and logging, an IR remote receiver for fast supervision of biological experiment and a simple local communication protocol via infrared proximity sensors to detect robots in short range.
\end{abstract}

Index Terms-Micro robots, mixed-society, animal machine interaction, perception

\section{INTRODUCTION}

Over the last decades, researchers in bio-inspired robotics have mimicked animals to design hardware and software structure of the robots. The RobotV [1], RHex [2], Biobot [3], HEL-roach [4] and the hexapod micro-robot [5] are examples of legged-robots which have been mechanically inspired by cockroaches.

Some researches have also developed hybrid robots by mixing the artificial and biological systems. The PheGMotIII [6] by Nagasawa et al. uses real cockroach antennas as a chemical sensor to follow pheromone tracks. Holzer [7] designed a system which controls the cockroaches' actuators by electric stimulation.

Instead of building exactly the same mechanism as animals, short-term goal of our work is to have robots which integrate into a society of animals, live inside the society and interact with them. Since the focus of our work is in collective-level, there is no need to have the same appearance as animals but the functionality of the robot must permit it to statistically produce the same collective behavior as animals e.g. to aggregate with the same probability distribution for duration of stay with respect to the number of animals around.

This means that not only the animals behavior is affected by the robots and other animals but also the robots behavior is affected by interactions with the animals and other robots in the collection. In fact every decision is made collectively by the whole system and a top-level observer of collective behaviors must not see any difference between the animal society and the mixed one. In our model the animal is thus considered as a black box and the important characteristics for our robot is to fit in the mathematical model of collective interactions among individuals involved in the group.

The long-term goal of the project is, once the robots are accepted to the society of animals, to manipulate the collective response of the society by modulating the behavioral parameters of robots. We hope then to propose guidelines towards a general methodology for performing such a control on mixed-societies.

Among the projects that are related to our work, there is the Robot Sheepdog [8] that controls a flock of ducks by moving them safely to a pre-determined position. Also, the W-M6 ratlike robot [9] by Ishii et al. tries to create a symbiosis between creature and robot by teaching a rat to push a lever to access a food source. These projects are different from what we are investigating in that their robots are not trying to integrate into the society. Instead they are trying to affect or supervise the society in a centralized manner.

Böhlen developed a robot [10] that interacts with three chickens in a cage. He manipulates some techniques to mechanically reduce chickens' anxiety towards moving machinery. The goal of the robot is to integrate with chickens but does not try to affect their behavior.

The present work is a part of the European project LEURRE, which aims to study mixed-societies of animals and robots. This multi-disciplinary project gathers the competence of biologists, ethologists, chemists and engineers coming from different European universities: Université Libre de Bruxelles, Université Paul Sabatier, Université de Rennes and Ecole Polytechnique Fédérale de Lausanne. The preliminary tests and developments are done with mixed-societies of cockroaches and robots.

In this project, our team is mainly involved in designing and building the robots and of course all tools that are needed to work efficiently with them. Another important task is to program the behaviors according to the models developed by the biologists. The resulting system is a useful toolbox for biological researches.

The current paper is organized as follows: first, the required functionality of our insect-like robot, the InsBot (reflected also in our previous paper [11]) is summarized in section II. Then we focus on the sensory devices for optimal environment detection, taking into account the hardware limitations of the 
robot (section III). It is followed by the architecture of the behavioral model (section IV). The test results (section V) are analyzed; finally conclusion and future works are explained.

\section{INSBOT DESCRIPTION}

A more detailed description of the robot is presented in [11], here comes a summary of the old design plus new updates and the detection methods.

As mentioned earlier, InsBot requirements do not specify that the robot should look like a real cockroach, but

- behaves like a real cockroach among their group.

- is accepted by cockroaches as a congener.

- is able to influence the global behavior of the cockroaches' society.

- is equipped with monitoring and debug facilities
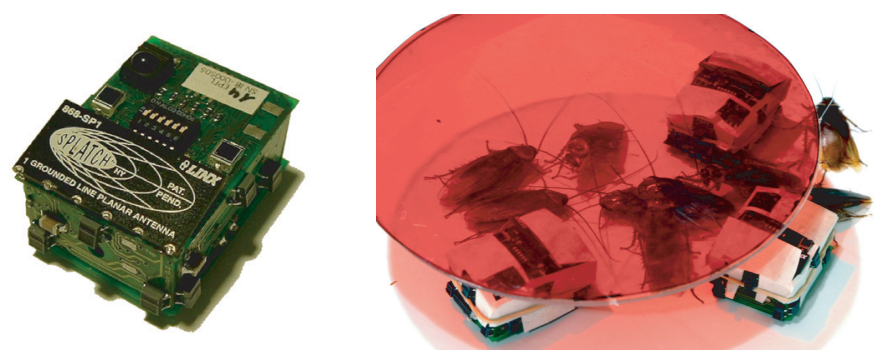

Fig. 1. Left: InsBot without cover. Right: InsBots with their paper covers aggregated with cockroaches under a shelter (Copyright ULB)

InsBot (Fig. 1) is a $41 \times 30 \times 19 \mathrm{~mm}^{3}$ robot. The rigid robot body is composed of PCBs which allow mechanical and electronic connections at once. It holds a $190 \mathrm{mAh}$ Li-Polymer battery that allows at least 3 hours autonomy required for the biological experiments and 2 miniature step motors for locomotion in differential drive configuration. A nail head is simply used as $3 \mathrm{rd}$ contact point. The robot weight is $17 \mathrm{gr}$ and it can reach speed of $4 \mathrm{~cm} / \mathrm{s}$.

\section{A. Sensors and Communication Tools}

In spite of its compact size, several sensors and communication tools are embedded in InsBot:

- 12 InfraRed (IR) proximity sensors. There are 3 sensors on each side of the robot placed at different heights to allow discrimination of different environment objects: cockroaches, walls and robots. They are also used for local communication between the robots.

- 2 photodiodes on top of the robots allow detection of the shelters.

- 1 linear camera in front of the robot, combined with IR sensors, helps to detect individual and group of cockroaches.

- 1 IR receiver to remote control the robot.

- 1 radio transceiver (at $868 \mathrm{MHz}$ ) to communicate with an external computer. This radio link is mainly used for debugging or monitoring purpose.

- 4 debugging LEDs which indicate the robot states.

\section{B. Control and Electronic Architecture}

The design includes two PIC18F6720 micro-processors as depicted in Fig. 2. Both processors have a $16 \mathrm{MHz}$ external clock, 128k program memory, 3840 byte of SRAM data memory and 1024 byte of ROM. The "Hardware Processor" is connected to most of the hardware resources. It prepares the sensory data by filtering the noise, scaling and calibrating their value. These information are then transmitted through a $400 \mathrm{kHz}$ I2C bus to the "Behavior Processor" that hosts the behavioral algorithm.

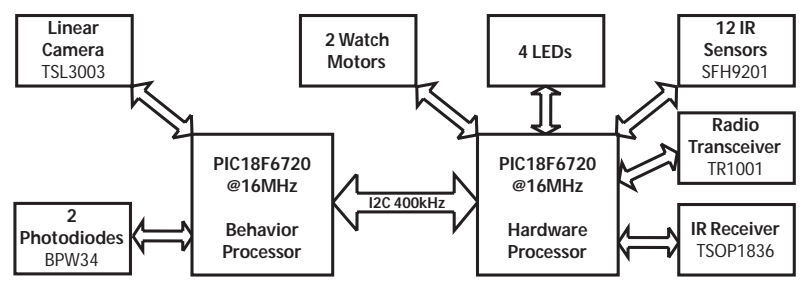

Fig. 2. Control and electronic architecture

\section{Pheromone cover}

To enhance the acceptance of the robot into the cockroach's colony, InsBot is covered by a paper that is impregnated with cockroaches' pheromone (Fig. 1 right side).

\section{InsBot Detection Methods}

In this section we focus on the detection algorithm that has been tuned for optimal environment perception. In fact, to behave like a real cockroach, the robot must first be able to detect the relevant features of the experimental setup which are the inputs of the behavior algorithm. These features are the living cockroaches, the setup walls, the shelters where cockroaches tend to rest and the other robots.

\section{A. The Experimental Setup}

As the InsBot sensing capabilities are limited by its lowpower small-size available sensors and mainly depend on the environment conditions, some details about the experimental setup are described (Fig. 3). Indeed the sensing and detection methods which are described in this chapter have been tuned to work in this particular environment.

The setup is a circular white plastic arena (1m diameter, $20 \mathrm{~cm}$ high) with an electrical fence to avoid the cockroaches standing up or escaping. The floor is composed of different layers which reduce the amount of vibrations that could potentially frighten the cockroaches. The ground white paper is changed after each experiment to avoid cockroaches being influenced by the remaining pheromone of the previous experiment. To avoid disturbance to the cockroaches' behavior and the robots' IR sensors, illumination is given by 4 neon light bulbs with low IR emission. Finally there are two circular suspended shelters under which cockroaches aggregate.

We now describe how the robot is able to distinguish the four main features of the setup: the 2 heterogeneous shelters, the living cockroaches, the arena walls and the other robots. 


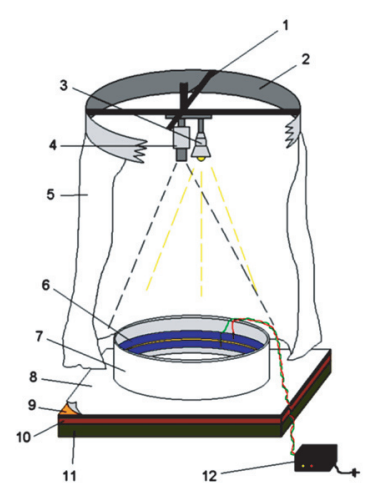

Fig. 3. Experimental setup composed of neon light (3), top camera (4), electrical fence $(6,12)$, white plastic arena $(7)$, paper layer $(8)$, phonic layer (9) and wooden layer (10). Shelters are not represented on the illustration (Copyright ULD)

\section{B. Shelter Detection}

The 2 different circular shelters (called "dark shelter" and "bright shelter" hereafter) are composed of dark plastic layers hanged at $5 \mathrm{~cm}$ from the ground. To create different levels of shadow different number of layers are grouped.

For detection and differentiation of both shelters, the light intensity is measured by 2 photodiodes mounted on top of the robot. Then their value is compared with thresholds computed during a calibration procedure at the beginning of the experiment. As the light intensity in the "center" of both shelters is distinct enough, the detection and differentiation quality is perfect.

There is a gradient of light from the center of the created shadow towards the borders with the maximum light intensity at the borders. Therefore some regions have the same light intensity under both shelters. In this case the robot treats them as the bright shelter. This induces no bias in the results since the problem also exists for real cockroaches.

\section{Cockroach Detection}

The cockroaches used in the mixed-society experiments are Periplaneta Americana (24-44mm long), shining red-brown which is a domiciliary species [12]. They have 6 legs for locomotion and 2 long (around $3 \mathrm{~cm}$ ) antennas for sensing. Due to the dark color of their body, they are hardly detectable by the robots IR sensors. But thanks to the sensors placement on the robot, a calibration procedure and some heuristic rules, they can be distinctively detected from $1.5 \mathrm{~cm}$ distance.

On each side of the robot there are 3 sensors (Fig.1). Two lateral sensors are close to the ground (called "bottom sensors" hereafter) and one is placed at center of each side with maximum height (called "top sensor" hereafter). Due to the cockroach's flat shape, the top sensors are less affected by the cockroaches than the bottom sensors as illustrated by left schematic of Fig.4. The solid curves on the right show the response of the IR sensors to a cockroach placed at different distances on right side of the robot. It shows that at far distances (more than $2.5 \mathrm{~cm}$ ) there is no response from the sensors, the cockroach is thus invisible. Between 2.5 to $1.5 \mathrm{~cm}$

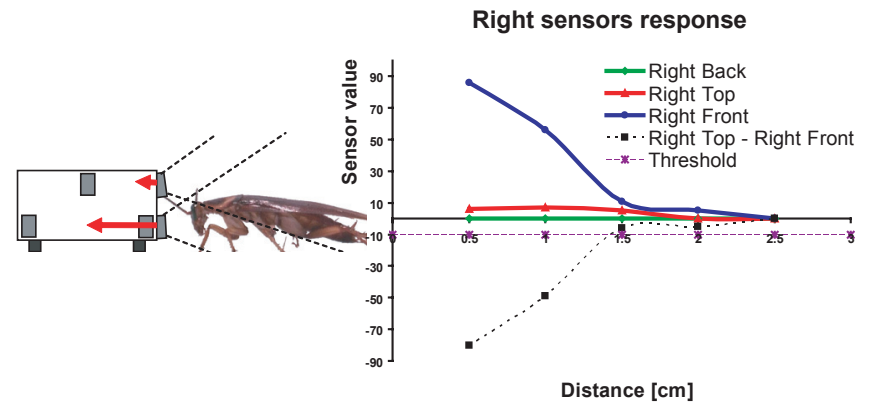

Fig. 4. On the left: IR sensors activated by the cockroach. On the right: IR sensors response when there is a cockroach on the right (front) side of the robot

the response of both sensors are almost equal, the cockroach is visible but not differentiable from a wall (See III-D for comparison). From $1.5 \mathrm{~cm}$ it is possible to detect cockroaches by thresholding the difference between top and bottom sensors (dashed curve of Fig.4).

Enhanced Cockroach Detection: There are some situations where IR sensors do not provide reliable information to well discriminate different objects, specially when a cockroach is located along the wall. As illustrated on Fig.5, the front left and rear left sensors would be activated even without the presence of the cockroach, because of the large aperture angle of the IR sensors. In this situation using the camera helps reducing misdetections of cockroaches.

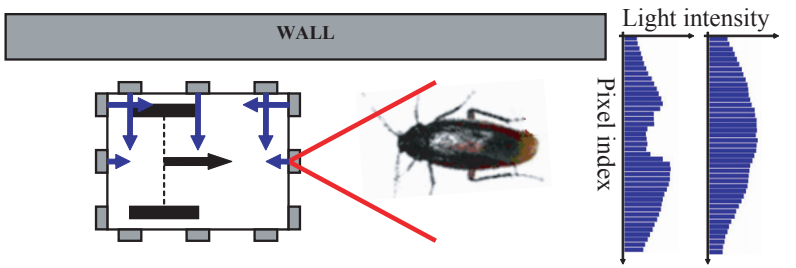

Fig. 5. IR sensors activation when the robot follows a wall and meets a cockroach: front left and rear left sensor are activated even without the presence of any cockroach. In red: camera field of view. On the right: linear camera response with and without any cockroach in front of the robot

Therefore cockroach detection on the front side where the risk of collision is the highest, is a combination of the IR sensing and linear camera processing. The algorithm for processing the linear camera detects discontinuities on the camera image (Fig.5 right side) due to presence of cockroaches.

\section{Wall Detection}

The wall of the circular arena is composed of a white plastic partially covered by a black colored electric fence starting from $3 \mathrm{~cm}$ height up to the top edges of the wall. So the visible part of the wall to IR sensors is $3 \mathrm{~cm}$ high. A simple schematic of the situation (Fig.6 left side) shows that the value of the top sensor should be close to the mean of the 2 others.

The solid curves on the right graphics (Fig.6) show the response of the right sensors when the robot is placed at different distances making a $45^{\circ}$ angle with the wall. It shows 

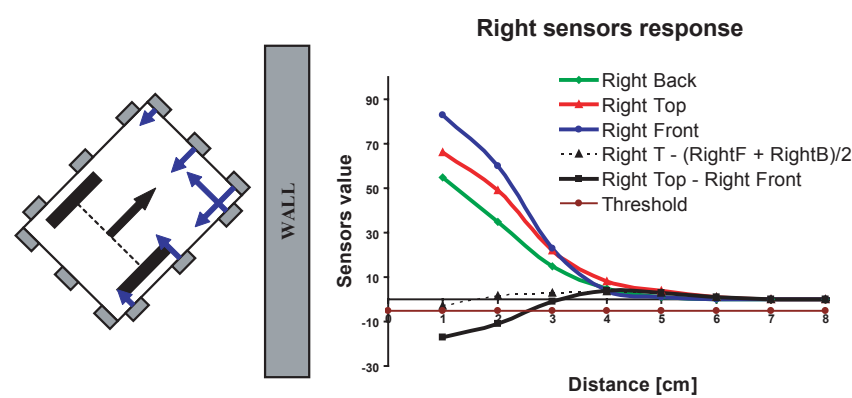

Fig. 6. On the left: IR sensors activated by the wall. On the right: IR sensors responses when there is a wall on the right side of the robot $\left(45^{\circ}\right.$ angle with the wall). For comparison with cockroach detection case: RightTop-RightFront curve is plotted

that at distances further than $7 \mathrm{~cm}$ there is no response from the sensors, the wall is invisible. Between 6.5 to $0 \mathrm{~cm}$ the wall is more and more visible with sensor values which confirm that the top sensor value is close to or bigger than the average of both bottom sensors (dashed curve represents the difference between the top IR sensor and the mean of the 2 bottom sensors). This graphics also shows that the difference between the top and bottom sensor (solid curve) is partially negative; this part overlaps with the cockroach case, that is why the cockroach detection threshold has been increased and the detection range is consequently decreased (Fig.4 for comparison)

\section{E. Robot detection}

Finally, InsBot must be able to distinguish the other InsBots from cockroaches and walls. An InsBot is currently considered as a cockroach, but for future experiments we would like the robot to react differently to cockroaches that to other robots. Only using IR sensors proximity values is not possible. Indeed the other robots look like a wall or a cockroach depending on their relative position as depicted in Fig.7. However a local communication protocol using IR sensors as transceiver has been implemented. Combining this information and the IR sensors proximity values allows distinguishing robots from cockroaches and walls.

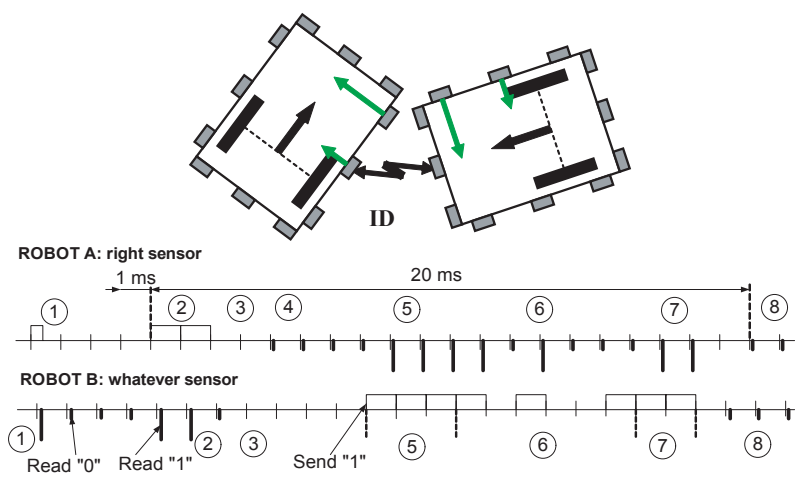

Fig. 7. Top: IR sensors response, when two robots meet could look like a cockroach as in this case. IR local communication protocol allows detecting and counting the robots. Bottom: chronogram of the communication protocol
Since IR sensors include an IR emitter and receiver, they can be used as transceivers as well as proximity sensors. Indeed if the robot receives IR signal while it has not emitted any signal, this means that another robot is emitting around (either for communication or proximity sensing purposes). This property has been used to establish a local communication protocol between nearby InsBots.

The protocol (Fig.7) includes a "hello/wakeup" signal (11) (2) to differentiate with the signal emitted when measuring proximity (1), that is similar to one bit (1). When this message is perceived by another robot (B), it becomes master and starts to send a message that start with 3 start bits (111 (5)). If the robot (A) which has sent the "hello/wakeup" signal receives these 3 start bit after a given time (3)+(4), it becomes slave and receives the message. The 8 bit communication messages include 6 data bits (6) and 2 stop bits (11) (7). These 6 data bits contain the unique ID of each robot. The behavior algorithm needs this ID to count the number of surrounding robots currently considered as cockroaches. For electronic and computational reason, communication is only implemented on the 4 top sensors.

\section{F. Calibration}

Due to several reasons a calibration phase should be repeated once every setup change: the inclination angle of proximity sensors is hard to adjust precisely. They are also not perfectly placed at the same height so they have different initial values. The floor paper and its flatness highly affect the bottom sensors. The illumination conditions vary in each experimental setup and the amount of light under each shelter changes as well. Orientation of shelters may vary for each experimental setup which changes the shelters gradient of light.

The calibration procedure developed for proximity sensors and shelters are activated via TV remote control upon the user requests. The computed calibration vectors are saved in the EEPROM and loaded after each restart. During regular process, these vectors are used to adjust the value of sensors and threshold the noise part.

\section{INSBOT BEHAVIOR}

The implemented behavioral model is a layered approach. Complex behaviors are decomposed into simpler behaviors. Behavior generation is divided into two parts: reactive behaviors (e.g. obstacle avoidance and wall following) are managed in hardware processor due to faster access to sensors and actuators. Higher level behaviors (e.g. aggregation) are implemented in the behavior processor.

\section{A. Reactive Behaviors}

Reactive behaviors are generated by means of potential field fusion method [14]. Each potential field assigns attraction or repulsion force (rx, ry) vector. The final movement direction and velocity corresponds to the resultant force of the weighted sum of these force vectors.

For example obstacle avoidance is the weighted sum of move-forward, cockroach-avoidance, robot-avoidance, wallavoidance, and unknown-object-avoidance. Another behavior 
like wall following which stands in higher layer is generated by weighted sum of obstacle-avoidance, right-wall-attraction, and left-wall-attraction. Weights are adjusted empirically for each behavior.

The resultant force ( $\mathrm{Rx}, \mathrm{Ry})$ is then converted to the speed of wheels (V1, Vr) by applying the following transformation:

$$
V l=R x+R y / 2 \quad V r=R x-R y / 2
$$

\section{B. High-level Behaviors}

Higher level behaviors like aggregation are implemented in the behavior processor. The mathematical model of the aggregation asks for a stochastic state machine which selects at each time step (here $500 \mathrm{~ms}$ ) the next action of the robot among \{move, turn, and stop\} action set (Fig.8). These actions are mapped to reactive behaviors in hardware processor. If robot is moving near periphery, move action means wall following behavior and turn means escaping from wall. In the center of the arena they mean regular obstacle-avoidance and regular turning respectively.

Actions are selected corresponding to a probability table which assigns a probability to each action based on the position of robot (center or periphery), shelter type (dark or bright), and number of cockroaches (or robots) around. The probability table is specified by extensive statistical data extraction on real cockroaches using visual tracking software and adapted to get similar behavior as the cockroaches or at least not disturbing their behavior.

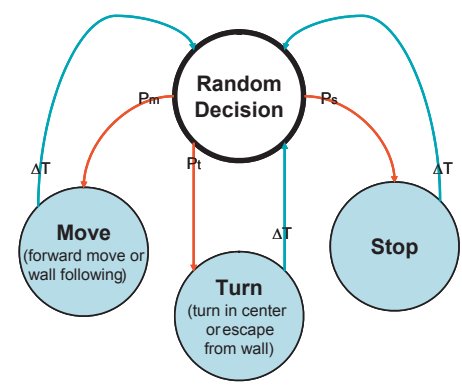

Fig. 8. The state machine for aggregation generation

\section{RESUlts}

Based on the discussed facts, we implemented a detection algorithm that uses the different responses of the top and bottom sensors, the local communication between robots and the linear camera response to distinguish cockroaches from arena walls and robots.

\section{A. Cockroach and wall detection}

Fig.9 displays the accuracy of the cockroach/wall detection algorithm implemented on the InsBot. These results were obtained by manually analyzing 900 different situations of a movie taken by the overhead camera and information of the wireless communication interface. For cockroach detection the distance is measured from the robot (body-border) to the closest point of the cockroach body (excluding legs and antennas).
These graphics confirm that cockroaches are visible from $2.5 \mathrm{~cm}$, but that optimal detection is only reached for cockroaches that are very close to the robot. The better performance in front side is due to introducing the linear camera to sensor fusion algorithms, the dashed curve represents the same result without the use of the linear camera. It is clear that the detection accuracy is close to detection in left/right side. This graphics also shows that walls are detectable at further distances than cockroaches with higher accuracy thanks to their better reflective properties.

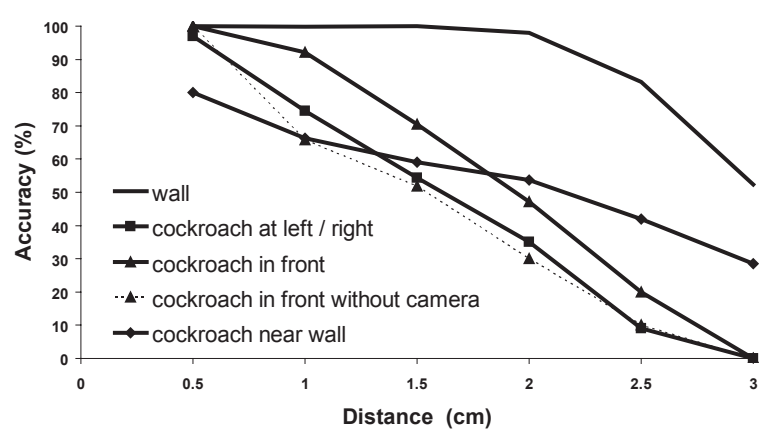

Fig. 9. Cockroach/wall detection accuracy vs. robot-cockroach/wall distance

The rather poor performance of cockroach detection at even short distances comes from several facts:

- Certain parts of the cockroach's body are less visible than others. The round compact head does well reflect IR signals, whereas the rear of its body composed of thin horizontal wings tends to reflect IR signals upwards.

- There are still some positions around the robot which are not well covered by IR sensors as depicted on Fig.10. The dashed shape indicates the regions where cockroaches activate the IR sensors (from $2.5 \mathrm{~cm}$ ) and the solid shape indicates the regions where cockroaches are effectively detected by the algorithm (from $1.5 \mathrm{~cm}$ ).

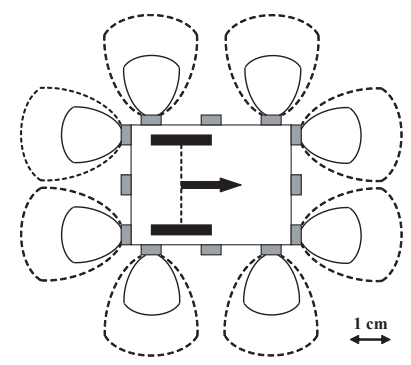

Fig. 10. Sensory coverage around robot: the dashed shape indicates the maximum range of bottom sensors activation by cockroaches (from $2.5 \mathrm{~cm}$ ) and the solid shape indicates the maximum range of cockroach recognition (from $1.5 \mathrm{~cm}$ )

\section{B. Robot detection}

Robot detection mainly depends on the local communication protocol reliability, that is rather difficult to characterize, because it depends on several variables. The communication rate depends on the distance between the two robots, obviously 
with higher rates when robots are closer. It also depends on the robots relative orientation: higher the overlap between the IR emitting and receiving cones is, higher is the communication rate.

Fig. 11 (left side) shows the communication rate between two robots: a robot is placed at different distances and with different orientations $\left(0^{\circ}, 45^{\circ}, 90^{\circ}, \ldots\right)$ to a fix robot (dark square in the center of the picture). For each position the number of received messages ( 8 bits including 2 stop bits) during a 30s. test was recorded.

Fig. 11 (right side) shows the percentage of correct messages among the received messages (Fig. 11 left side). These results are rather good: even in situations where communication is difficult (lower communication rates), the percentage of correct messages is rather high (between 70-100\%).

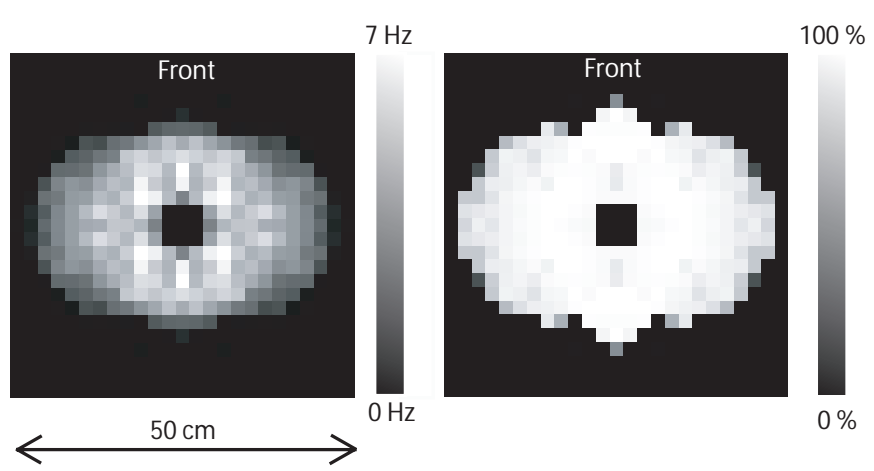

Fig. 11. Local communication between 2 robots. Left: communication rate. Right: communication success rate

Robot detection range is obtained by combining this local communication data with the IR proximity values.

\section{CONCLUSION AND FUture WORKS}

Details on the perception and behavior implementation of the miniature robot InsBot are explained in this paper. Due to limitation in the size of the robot and long-time autonomy needed for biological experiments with cockroaches, the hardware parts and processing algorithm have been highly optimized. Different problems arisen from imposed simplifications and limited sensory information are explained and the solutions described.

The sensor fusion methods combined with heuristic rules that came from our knowledge about the experimental setup allowed the robots having good discrimination among different objects in the environment. Cockroaches and walls are detected by using the infra-red proximity sensors mounted in different heights all around the robot. To have less collisions and thus a more friendly behavior with cockroaches, a linear camera was introduced on the front side of the robot that enhanced the detection quality. A simple local-range communication protocol through infra-red sensors was established for robot detection.

However more investigation should be done to completely solve some raised problems. Using local communication for robot detection introduces noise on the sensors of other surrounding robots, mainly due to the periodically "hello/wakeup" signals that robots emit to show their presence. This noise does of course disturb detection. We are working on appropriate filters to reduce it. The cockroach and wall detection characterizations given in this paper do not consider the presence of another surrounding robot that could potentially disturb detection due to these local communication signals. Moreover, when there are a lot of objects around the robot, the information from IR sensors get more and more difficult to interpret.

Finally the biological experiments showed that the robots are accepted by groups of cockroaches and that the mixedsociety of robots and cockroaches has statistically close behavior as a pure cockroach society. The results of these biological experiments will be submitted very soon to biology conferences.

\section{ACKNOWLEDGMENT}

The LEURRE project [13] is funded by the Future and Emerging Technologies programme (IST-FET) of the European Community, under grant IST-2001-35506. The information provided is the sole responsibility of the authors and does not reflect the Community's opinion. The Community is not responsible for any use that might be made of data appearing in this publication. The Swiss participants to the project are supported under grant 01.0573 by the Swiss Government.

\section{REFERENCES}

[1] D.A.Kingsley, R.D.Quinn, R.E.Ritzmann, A Cockroach Inspired Robot With Artificial Muscles, Proceedings of the 2nd International Symposium on Adaptive Motion of Animals and Machines, Kyoto, 2003.

[2] U.Saranli, M.Buehler, D.E.Koditscheck, RHex - A Simple and Highly Mobile Hexapod Robot, International Journal of Robotics Research, Vol. 20, No.7, pp616-631, 2001.

[3] F.Delcomyn, M.E.Nelson, Architectures for a biomimetic hexapod robot, Robotics and Autonomous Systems 30, pp5-15, 2000.

[4] N.Kagawa, H.Kazerooni, Biomimetic Small Walking Machine, International Conference on Advanced Intelligent Mechatronics Proceedings, Como, 2001.

[5] Y.Guozheng, D.Yi, A Novel Biomimetic Hexapod Micro-robot, International Symposium on Micromechatronics and Human Science, 2002.

[6] S.Nagasawa, R.Kanzaki, I.Shimoyama, Study of a Small Mobile Robot that uses Living Insect Antennae as Pheromone Sensors, Proceedings of the 1999 International Conference on Intelligent Robots and Systems, 1999.

[7] R.Holzer, I.Shimoyama, Locomotion Control of a Bio-Robotic System via Electric Stimulation, Proceedings of the International Conference on Intelligent Robots and Systems, 1997.

[8] R.Vaughan, N.Sumpter, J.Henderson, A.Frost, S.Cameron, Robot Control of Animal Flocks, Proceedings of the IEEE ISIC Joint Conference, 1998.

[9] H.Ishii, M.Nakasuji, M.Ogura, H.Miwa, A.Takanishi, Accelerating Rat's Learning Speed Using a Robot - The robot autonomously shows rats its functions, Proceedings of the 2004 International Workshop on Robot and Human Interactive Communicaiton, Kurashiki, Okayama Japan, 2004.

[10] R.Böhlen, A robot in a cage, Proceedings IEEE International Symposium, 1999

[11] A.Collot, G.Caprari, R.Siegwart, InsBot: Design of an Autonomous Mini Mobile Robot Able to Interact with Cockroaches, Proceedings of the International Conference on Robotics and Automation, New Orleans, pp2418-2423, 2004.

[12] Bell, W.J. and Adiyodi, K.G., The American Cockroach, London : Chapman and Hall Ltd, 1982.

[13] LEURRE Project official website, http://leurre.ulb.ac.be.

[14] R.C. Arkin, Behavior-based Robotics, MIT Press, 1998. 